\title{
Phonoechocardiography and intracardiac phonocardiography in hypertrophic cardiomyopathy
}

\author{
James A. Shaver, Roando F. Alvares, P. Sudhakar Reddy and Rosemarie Salerni \\ University of Pittsburgh School of Medicine, Division of Cardiology, Pittsburgh, PA 15261, USA.
}

\begin{abstract}
Summary: The salient phonoechocardiographic features of patients having hypertrophic cardiomyopathy (HCM) with or without left ventricular outflow tract (LVOT) gradients are reviewed. Intracardiac sound and pressure recordings from high fidelity catheter-tipped micromanometers have documented that the precordial murmur is the summation of both the systolic ejection murmur (SEM) arising from the LVOT, as well as the mitral regurgitant murmur recorded from the left atrium. The intensity of the precordial murmur varies directly with the LVOT gradient, which in turn is determined primarily by the contractility and loading conditions of the left ventricle.

Reversed splitting of the second heart sound $\left(\mathrm{S}_{2}\right)$ with paradoxical respiratory movement is a common finding in HCM, and when present, almost always denotes a significant LVOT gradient. It is due to marked lengthening of the left ventricular ejection time secondary to prolongation of the contraction and relaxation phases of left ventricular systole. The presence of a fourth heart sound $\left(S_{4}\right)$ is the rule in $H C M$ when normal sinus rhythm is present, and is a reflection of a forceful left atrial contraction into a hypertrophied noncompliant left ventricle. A third heart sound $\left(\mathbf{S}_{3}\right)$ is also common in $\mathrm{HCM}$, and often the initial vibrations occur before the 0 point of the apexcardiogram (ACG) and continue giving the auscultatory impression of a diastolic rumble. When associated with a loud $S_{1}$, which is frequently present, the clinical presentation may mimic mitral stenosis. This is particularly true when the patient has chronic atrial fibrillation. Careful attention to evidence of marked left ventricular hypertrophy as well as the typical echocardiographic findings of HCM preclude this diagnosis.

In conclusion, phonoechocardiography is a simple non-invasive technique which almost always makes the definitive diagnosis of HCM.
\end{abstract}

Phonocardiography is the graphic representation of the bedside cardiovascular examination. It includes not only the recording of the auscultatory phenomena of the precordium, but also the carotid, venous, and cardiac apical pulsations simultaneously with the electrocardiogram (Shaver, 1981). When coupled with the M-mode echocardiogram, the diagnostic capabilities of this technique are further enhanced by correlating the sound and pressure tracings of the cardiac cycle with dynamic valvular and ventricular wall motion. Probably no disease lends itself so well to evaluation by these techniques as hypertrophic cardiomyopathy (HCM), because of its unique physical and anatomical findings. In this report, the salient features of patients with HCM will be described as recorded by phonoechocardiography. Furthermore, new insights into the mechanisms of the auscultatory findings in this entity will be provided by utilizing intracardiac recordings of sound and pressure

Correspondence: J.A. Shaver, M.D. obtained from high fidelity catheter-tipped micromanometers.

The phonocardiogram of a 54 year old patient with HCM is shown in Figure 1, and is typical of patients with this disease having a significant left ventricular outflow tract gradient. At cardiac catheterization, a resting peak gradient of $60-80 \mathrm{mmHg}$ was found with a left ventricular peak pressure of $180 \mathrm{mmHg}$, and a left ventricular end-diastolic pressure of $16 \mathrm{mmHg}$. The carotid pulse has the typical rapid rise followed by a late systolic plateau, and the left ventricular ejection time is prolonged. The apexcardiogram (ACG) demonstrates the classic triple pulsation consisting of the presystolic a wave, an early systolic outward pulsation, and a late systolic bulge. A loud fourth heart sound $\left(S_{4}\right)$ occurs during the rapid upstroke of the a wave, and a softer third heart sound $\left(S_{3}\right)$ occurs during the rapid filling wave (RFW) of the ACG. A grade V systolic ejection murmur begins during early left ventricular ejection shortly after the development of the left ventricular-aortic pressure gradient, and peaks during mid-systole at the time of the peak gradient. 


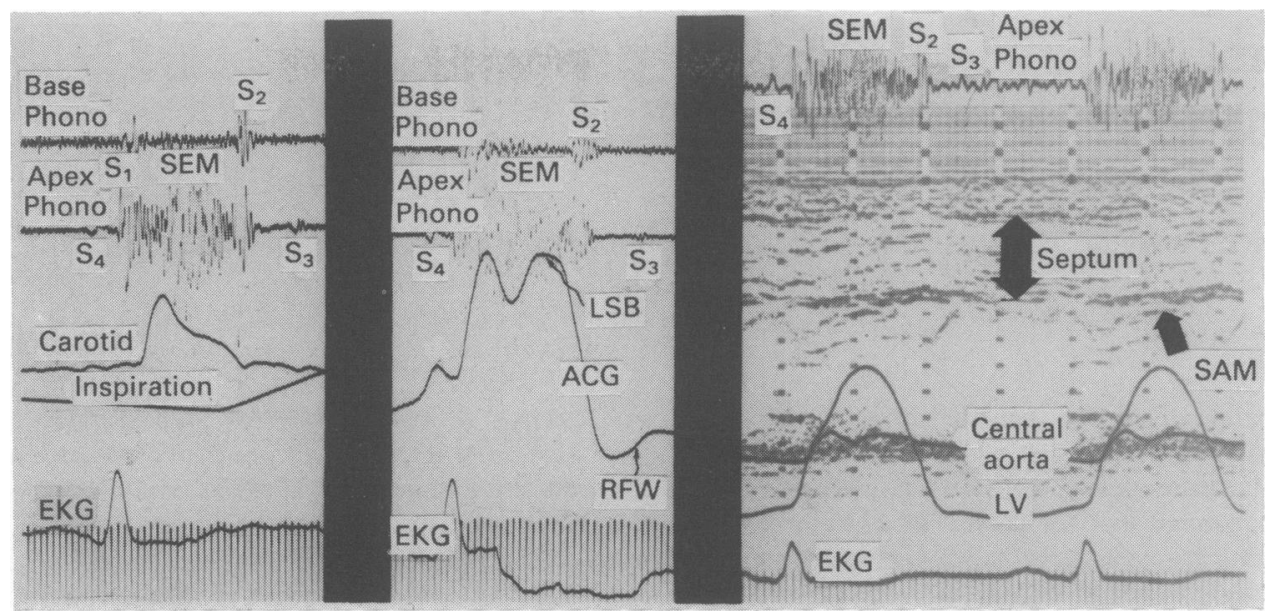

Figure 1 Simultaneous base and apex phonocardiograms are recorded with the carotid pulse and apexcardiogram (ACG) in the left and centre panels respectively, in a 54 year old male with HCM. The carotid pulse rises rapidly, has a late systolic plateau, and a prolonged ejection period. Prominent $S_{4}$ and $S_{3}$ are demonstrated, and are associated with the a wave and the rapid filling wave (RFW) respectively, of the ACG. Note the late systolic bulge (LSB) on the ACG. The second heart sound $\left(S_{2}\right)$ is single. A loud grade $V$ systolic ejection murmur (SEM) is present, and is of greatest intensity at the apex. In the right panel, the apical SEM is recorded together with the M-mode echocardiogram. Simultaneous high fidelity left ventricular and central aortic pressures are recorded by cathetertipped micromanometers. Marked thickening of the interventricular septem and systolic anterior motion (SAM) of the mitral valve are present on the echocardiogram. A large systolic pressure gradient is demonstrated, beginning shortly after the onset of SAM.

Systolic anterior motion (SAM) of the mitral valve is present, and its duration correlates well with the duration and magnitude of the pressure gradient (Pollick et al., 1984).

Frequently on auscultation, the skilled observer has difficulty in deciding whether the systolic murmur is ejection or regurgitant in nature. The explanation for this confusion is readily understood by analysis of the intracardiac sound and pressure recordings shown in Figure 2, as recorded from another patient with HCM. A typical systolic ejection murmur is recorded from the catheter-tipped micromanometer in the left ventricular outflow tract, while a late systolic murmur of mitral regurgitation is recorded from the transseptal left atrial micromanometer. The onset of this latter murmur begins shortly before contact of the anterior mitral leaflet with the septum (SSC) as timed by simultaneous M-mode echocardiography of the mitral valve. Thus, the resulting external murmur recorded by the precordial phonocardiogram is the summation of both murmurs as transmitted to the chest wall.

In patients with dynamic left ventricular outflow tract gradients, the intensity of both the systolic ejection murmur and the mitral regurgitation murmur varies directly with the level of the pressure gradient. Thus, physiological manoeuvres and pharmacological interventions which increase the pressure gradient will increase the intensity of the precordial murmur and? vice versa. Decreases in left ventricular preload and afterload, or increases in left ventricular contractility are associated with increases in both the pressure gradient and the intensity of the murmur, while increases in left ventricular preload and afterload or decreases in left ventricular contractility will decrease the pressure gradient and the intensity of the murmur (Glancy et al., 1971; Reddy et al., 1971). For example, both the upright posture and the strain phase of the Valsalva manoeuvre decrease venous return and left ventricular preload, and the murmur increases in intensity. Upon reclining or with prompt squatting, augmented venous return increases left ventricular preload, and the murmur decreases in intensity. Vasodilator drugs, e.g. inhaled amyl nitrate, decrease blood pressure and a marked increase in the intensity of the murmur occurs, whereas vasoconstrictive drugs, such as infused phenylephrine, increase the afterload and the murmur is decreased or abolished. Inotropic drugs such as digitalis and catecholamines increase the intensity of the murmur while beta blockade usually decreases it. The responses to these interventions are relatively specific for HCM, although occasionally patients with a mitral valve prolapse syndrome behave similarly. However, the clinical setting of this entity is easily differentiated and rarely confused with HCM. 


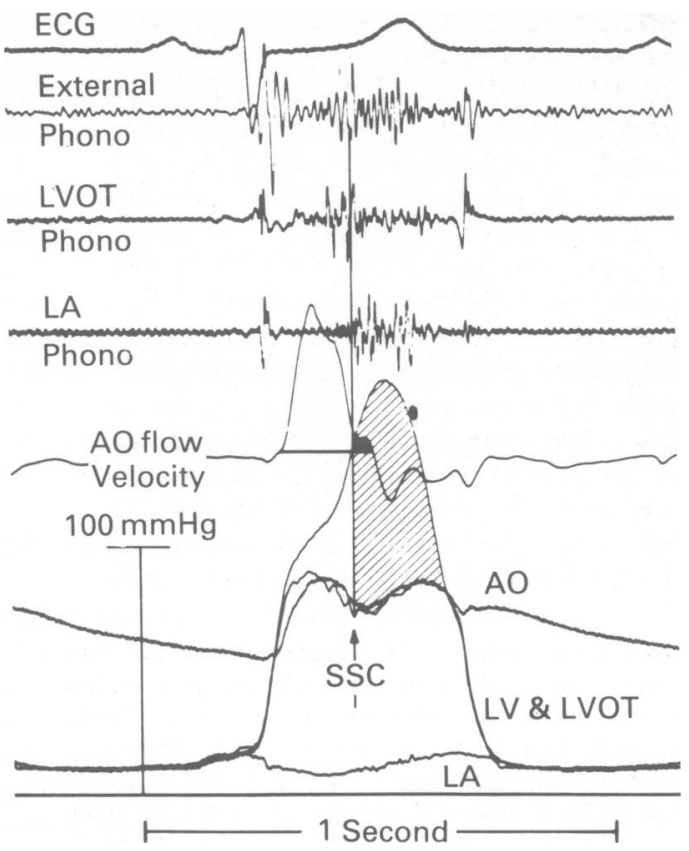

Figure 2 Catheter-tipped micromanometer pressures are recorded from the left atrium (LA), left ventricle (LV), left ventricular outflow tract (LVOT), and aorta (AO), together with external sound and intracardiac sound recorded from micromanometers in the LA and LVOT, in a patient with HCM. Aortic flow velocity is recorded by a flow probe in the central aorta. The vertical line denotes the time of SAM-septal contact (SSC) as determined by simultaneous M-mode echocardiography of the mitral valve. Note that the majority of aortic flow occurs before SSC. A SEM is recorded from the LVOT, and a late systolic mitral regurgitant murmur is recorded from the LA. The external phonocardiogram represents the summation of these two murmurs. (Courtesy of Dr Joseph Murgo, San Antonio, Texas).

In Figure 3, a finding unique to $\mathrm{HCM}$ with a resting or provocable gradient is shown. Following the long diastolic pause after a premature ventricular contraction (PVC), there is a significant increase in the intensity of the left ventricular outflow murmur associated with a decreased pulse pressure in the central aortic tracing. The normal response after a long filling cycle would be an increase in the intensity of any systolic ejection murmur associated with an increase in the arterial pulse pressure. The finding of a decreased pulse pressure following a long diastolic pause can be easily appreciated by palpating the carotid pulse at the bedside when extra systoles are present, and is diagnostic of this condition. The increase in the intensity of the externally recorded murmur following the compensatory pause after the

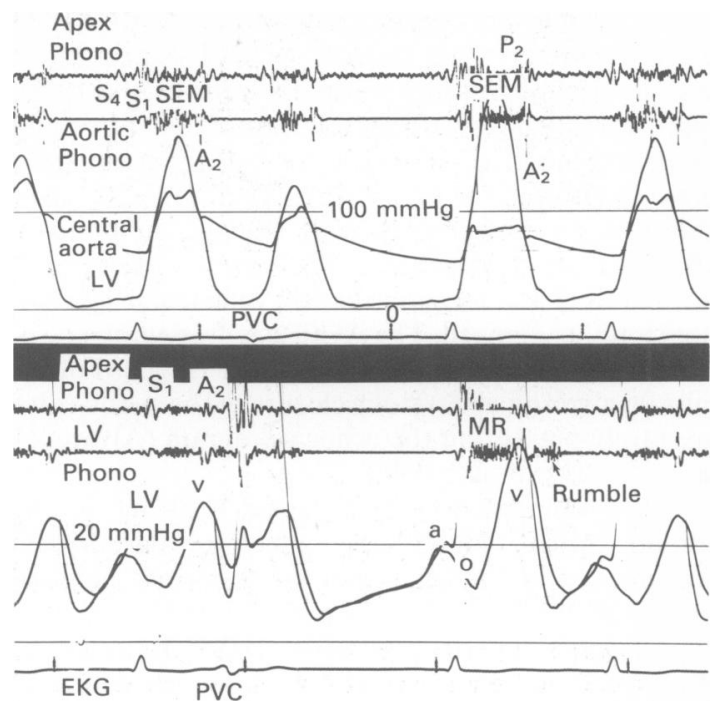

Figure 3 Top panel: Left ventricular and central aortic catheter-tipped micromanometer pressures are recorded, together with the apex phonocardiogram and intracardiac sound from the central aorta in a 71 year old woman with HCM having a dynamic LVOT gradient. Following a long diastolic filling period after a premature ventricular contraction (PVC), there is a marked increase in the LVOT gradient associated with a significant increase in the SEM, as recorded externally and in the central aorta. This is concomitant with a decrease in the pulse pressure recorded in the central aorta. In the lower panel, LV and LA pressures are recorded from equisensitive catheter-tipped micromanometers, together with the apex phonocardiogram and intracardiac sound from the inflow tract of the LV. A mitral regurgitant murmur is recorded from the $L V$ phono, while the left atrial $V$ wave rises to a peak of $20 \mathrm{mmHg}$. Following the compensatory pause after the PVC, there is a marked increase in the intensity of the mitral regurgitant murmur recorded by the $L V$ micromanometer with a left atrial $V$ wave peaking to $40 \mathrm{mmHg}$. Note the persistent true early diastolic gradient present in all complexes, and the concomitant early diastolic rumble recorded by the LV inflow phonocardiogram.

premature contraction is due not only to an increase in the left ventricular outflow ejection murmur, but also due to an increase in the degree of mitral regurgitation in the beat following the premature contraction. This is nicely shown in Figure 3 by an increase in the height of the left atrial $\mathrm{V}$ wave and by an increase in the intensity of the mitral regurgitant murmur as recorded by the intracardiac micromanometer. Again, the increased intensity of the precordial murmur is a summation of both the systolic ejection murmur and the mitral regurgitant murmur.

In the absence of a left ventricular outflow gradient 
at rest or with provocation, the murmur of HCM is much less impressive (Figure 4). Although a short systolic ejection murmur is usually recorded due to the rapid early left ventricular ejection, it is softer and extends through less of systole than when a gradient is present (Murgo et al., 1980). There is also little variation in its intensity with changes in preload, afterload, or contractility. The carotid upstroke is normal in character, and the left ventricular ejection time is not prolonged. The ACG usually demonstrates a prominent a wave associated with an $\mathrm{S}_{4}$. SAM of the mitral valve and midsystolic closure of the aortic valve are rarely present on the echocardiogram (Alvares $e t$ al., 1984; Doi et al., 1980).

The documentation of reversed splitting of the second heart sound $\left(\mathbf{S}_{2}\right)$, having paradoxical movement with respiration, is an important physical finding in HCM. In the 64 patients with idiopathic hypertrophic subaortic stenosis (IHSS) reported by Braunwald and associates (1964), it was present in 22 patients (34\%), and was associated with a significantly higher gradient than in those patients with a normal or single $S_{2}$. In a recent study by Alvares and associates (1984), 15 of 84 patients with HCM were studied by phonoechocardiography and were documented to have reversed splitting. Catheterization data was available in 13 of these patients, and in all a significant gradient was present at rest or with provocation. In addition, all 15 patients had systolic anterior motion documented by echocardiography, and 14 of 15 also had midsystolic closure of the aortic valve. The mechanism responsible for the delayed aortic closure sound $\left(A_{2}\right)$ is marked prolongation of the left ventricular ejection time resulting from a combination of prolongation of the contraction and relaxation phases of left ventricular systole (Figure 5) (Alvares et al., 1984). When lesser degrees of prolongation of ejection time are present, the $S_{2}$ may be single (Figure 1) or splitting may be physiological (Figure 4).

When normal sinus rhythm is present, an $\mathrm{S}_{4}$ is the rule in HCM (Figures 1, 4, 6). It is the result of a forceful left atrial contraction into a noncompliant left ventricle (Goodwin, 1982; Reddy et al., 1971). Since massive left ventricular hypertrophy is present in patients with or without left ventricular outflow gradients, the $S_{4}$ is common in both situations. As shown in Figures $1,4,6$, the $S_{4}$ is the auscultatory counterpart of the presystolic apical pulsation occur-

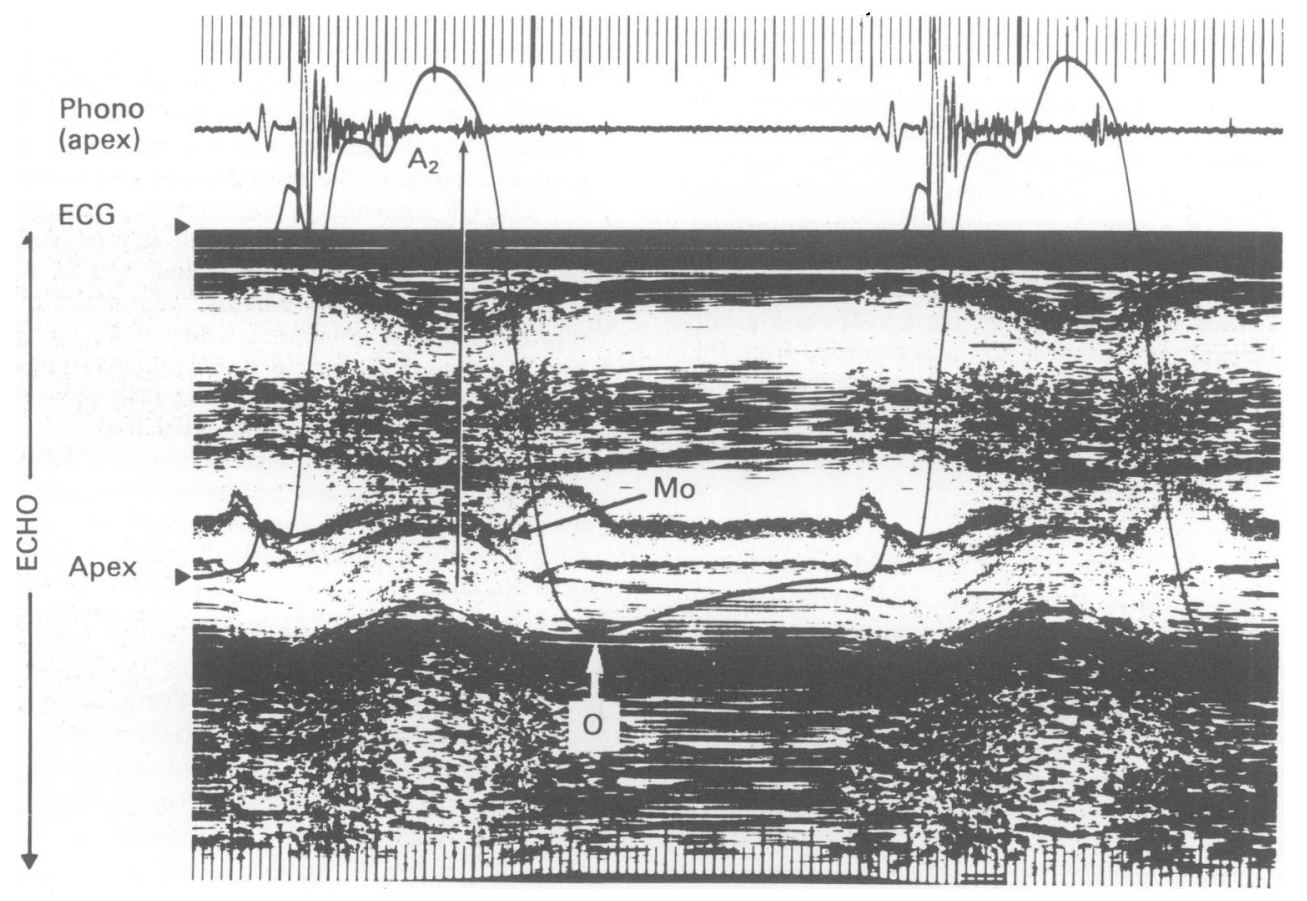

Figure 4 Simultaneous ACG and M-mode echocardiogram are recorded together with the ECG in a 41 year old male with HCM. A short SEM is present. A prominent presystolic pulsation is demonstrated on the ACG, which is coincident with the loud $S_{4}$ on the apex phonocardiogram. Splitting of $S_{2}$ is normal, and marked prolongation of the isovolumic relaxation period $\left(A_{2}-M O\right)$ to $110 \mathrm{~ms}$ is demonstrated. SAM of the mitral valve is absent. Marked thickening of the septum is present, with asymmetric hypertrophy. No LVOT gradient was measured at cardiac catheterization. (From Alvares et al., 1984, reproduced by permission). 


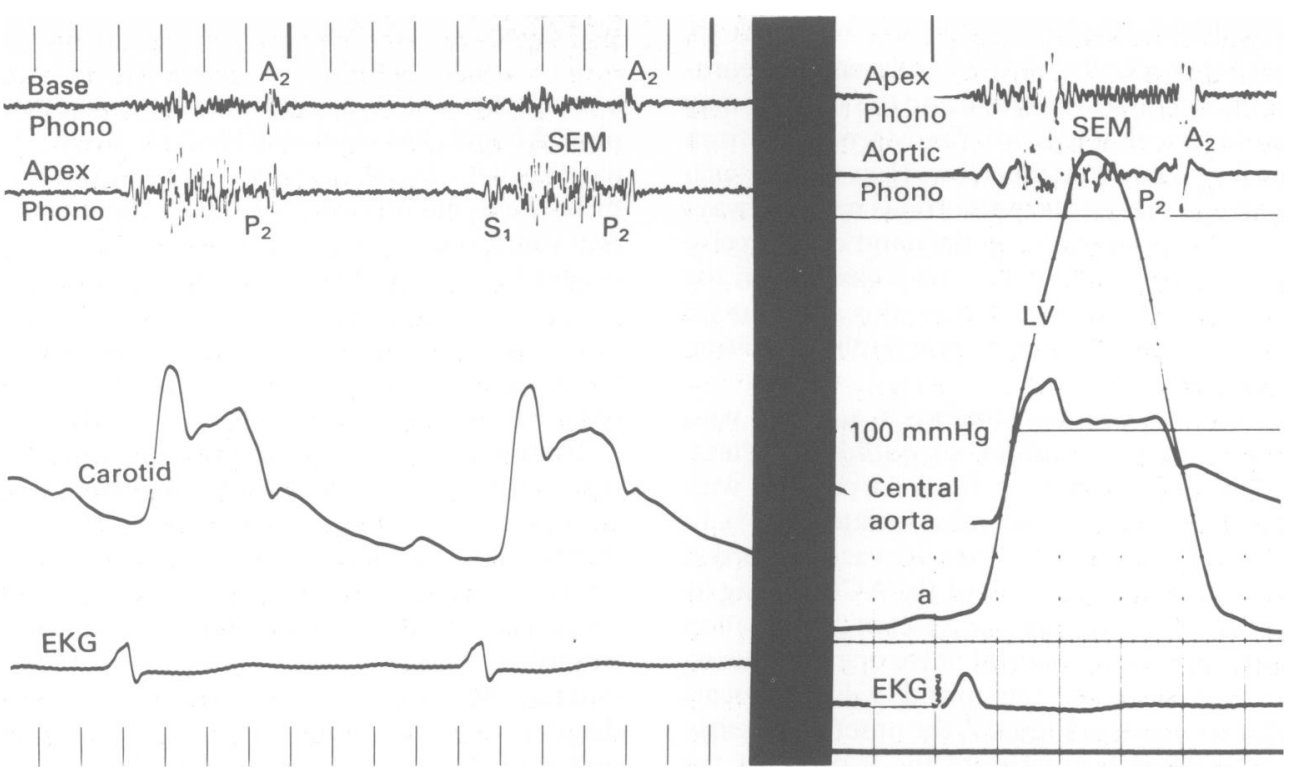

Figure 5 Left panel: Reversed splitting of $\mathrm{S}_{2}$ is documented by the simultaneous recording of the carotid pulse with the base and apex phonocardiograms in a 71 year old woman with HCM. Right panel: A large resting LVOT gradient was measured with equisensitive catheter-tipped micromanometers in the $\mathrm{LV}$ and central aorta. $\mathrm{A}_{2}$ is markedly delayed, due to both prolonged LV contraction and relaxation (same patient as Figure 3 ).
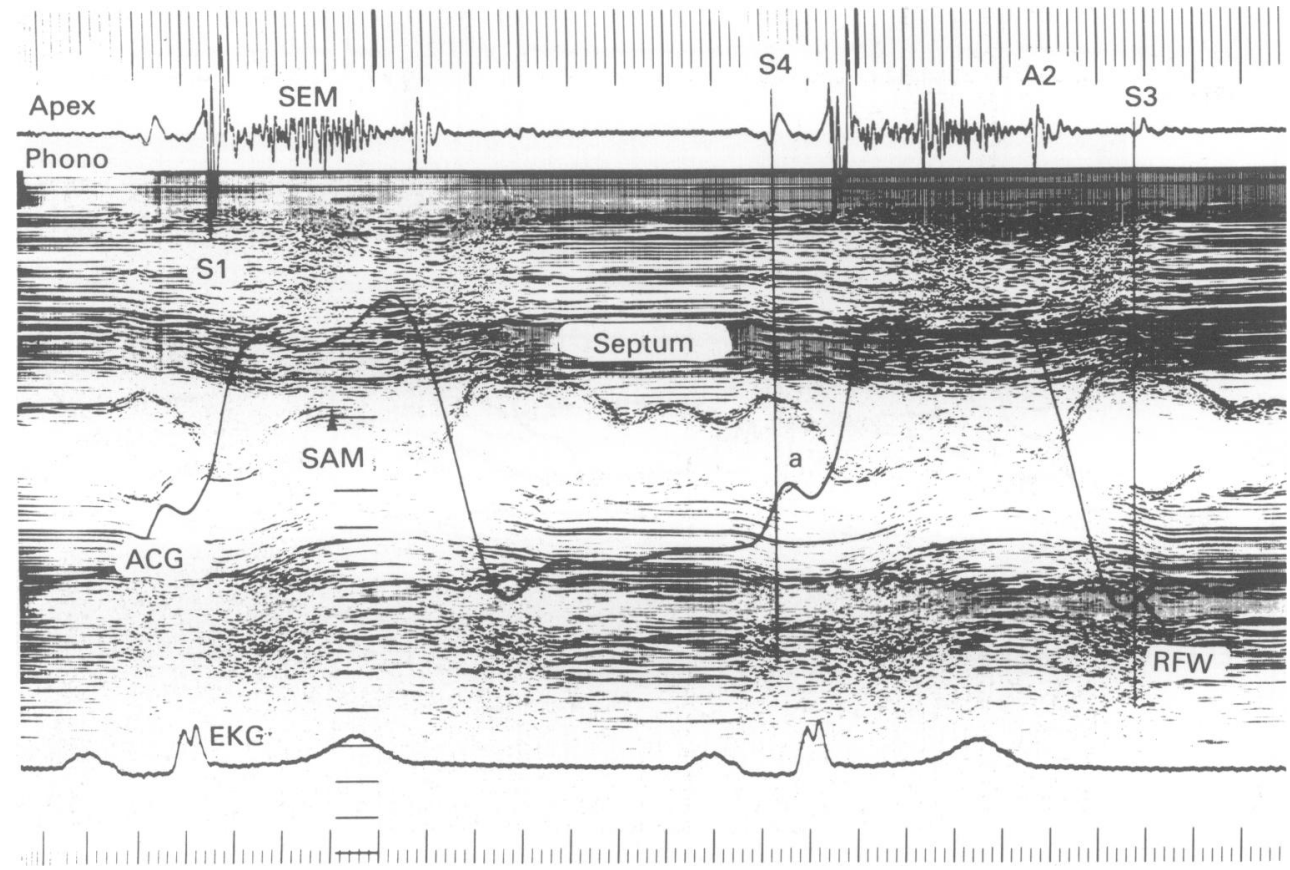

Figure 6 Simultaneous apex phonocardiogram, ACG, M-mode echocardiogram and ECG are recorded in a patient with $\mathrm{HCM}$, having both $\mathrm{S}_{4}$ and $\mathrm{S}_{3}$. The $\mathrm{S}_{4}$ occurs shortly after the maximal opening of the atrial wave of the mitral valve echocardiogram during the rapid upstroke of the a wave of the ACG. The $S_{3}$ occurs 80 ms after the maximal opening of the mitral valve during the RFW of the ACG. Note the late systolic bulge demonstrated on the ACG. A prominent SEM is recorded, and SAM of the mitral valve is observed. 
ring during the rapid rise of the a wave of the ACG. Although easy to identify by the simultaneous recording of the apex phonocardiogram and the ACG, it may be difficult to auscult if it is not clearly separated from $S_{1}$, or if its frequency content is low. However, in such cases, the apical presystolic pulsation is nearly always present and easy to palpate, as the hand can perceive low frequency vibrations much more easily than the ear. When atrial fibrillation occurs, this sound is no longer present, and the apical presystolic pulsation likewise disappears.

The $S_{3}$ is also a common finding in patients with HCM, either with or without an outflow gradient. Such a sound was present in 38 of 64 patients with IHSS reported by Braunwald and associates (1964). In Figures 1 and 6, typical $S_{3}$ gallops are easily recorded and occur well after the 0 point of the ACG during its RFW. As shown in Figure 6, the onset of the $S_{3}$ gallop occurs after the maximal mitral valve opening during the early E-F slope of the mitral valve echocardiogram. In contrast, in Figure 7, the onset of the early diastolic vibrations begin before the 0 point of the ACG, and on close inspection, continue for considera- ble duration, resembling more an early diastolic rumble rather than a discrete low frequency sound. In a comprehensive study utilizing phonoechocardiography, Alvares has demonstrated an $S_{3}$ in 19 of 60 patients with HCM in normal sinus rhythm; in 10 of these the $S_{3}$ gallop occurred before the 0 point of the ACG and often appeared as a rumble, as shown in Figure 7 (Alvares, 1980). Of further interest, he noted that an $S_{3}$ was present in 8 of 9 patients with HCM having atrial fibrillation, and in all it occurred before the 0 point of the ACG, often having vibrations of considerable duration.

The presence of such diastolic rumbles have been reported by other investigators in patients with $\mathrm{HCM}$ with clinical findings which mimic mitral stenosis (Shabetai \& Davidson, 1972; Smith et al., 1975). However, as pointed out by these reports, careful attention to evidence of marked left ventricular hypertrophy, as well as the typical findings of the echocardiogram allow the clinician to readily make the correct diagnosis of HCM, even when the loud $S_{1}$ and diastolic rumble clinically simulate mitral stenosis. It is possible that such diastolic rumbles may be secondary to

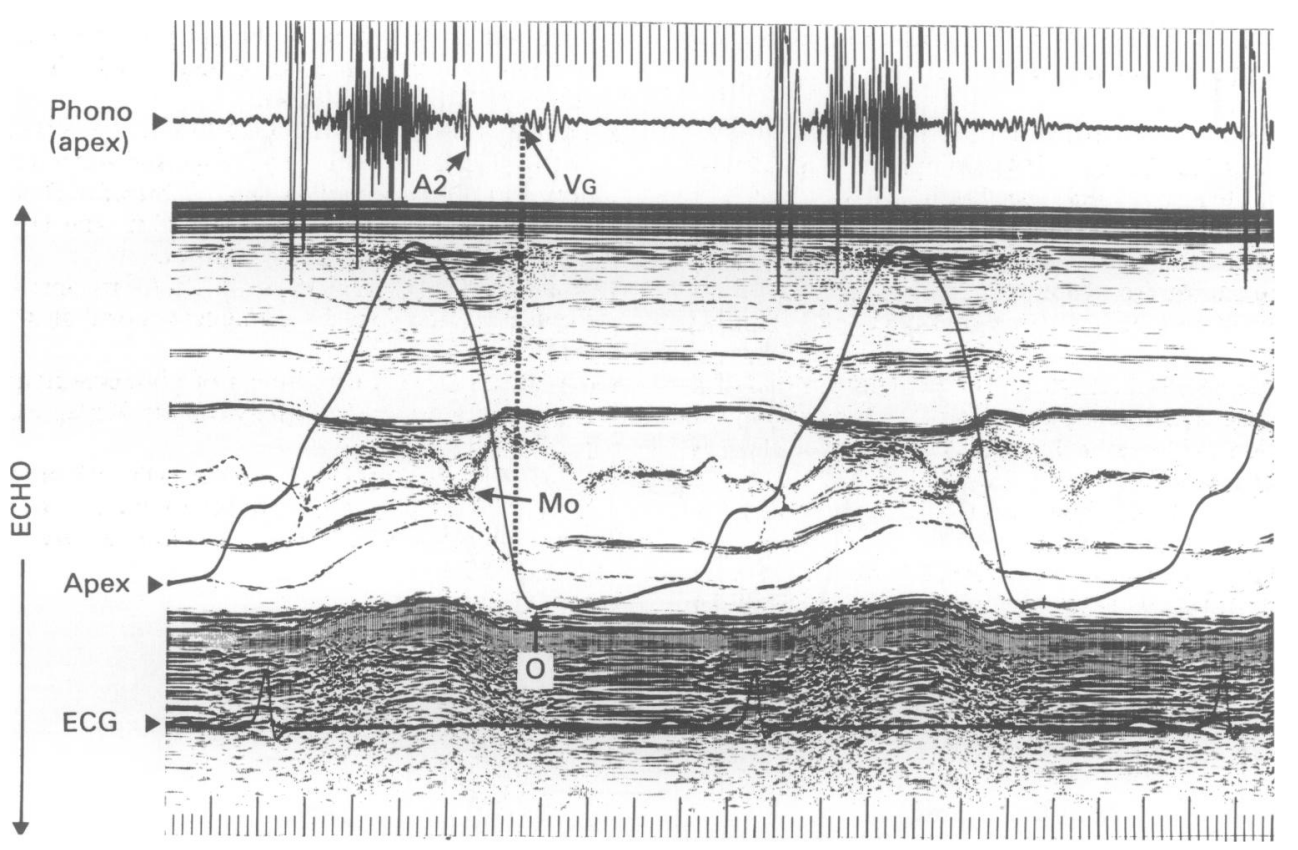

Figure 7 Simultaneous apex phonocardiogram, ACG, and M-mode echocardiogram are recorded together with the ECG in a patient with HCM having prominent early diastolic vibrations occurring well before the 0 point of the ACG. The first heart sound $\left(S_{1}\right)$ is loud, and together with the early diastolic apical rumble, may mimic mitral stenosis on auscultation. However, the prominent presystolic heave and sustained apical systolic pulsation are incompatible with this diagnosis. The echocardiogram unequivocally documents the diagnosis of HCM with massive septal hypertrophy, SAM of the mitral valve, and reduced LV cavity size. A prominent SEM is recorded typical of HCM with a significant LVOT gradient, which was documented at sardiac catheterization. (From Alvares, 1980, reproduced by permission). 
increased impedance to left ventricular filling secondary to decreased diastolic compliance of the left ventricle. That such a phenomenon may exist is suggested by the ability to record a diastolic rumble in the inflow tract of the left ventricle during early diastolic filling, when a definite left atrial-left ventricular pressure gradient is demonstrated by equisensitive catheter-tipped micromanometers (Figure 3). In this patient, there was no clinical or echocardiographic evidence of mitral valve stenosis, although the LV cavity was small with marked hypertrophy of the septal and posterior walls and prominent SAM of the mitral valve was evident. As shown in Figures 3 and 5 , neither a typical $S_{3}$ nor an $S_{3}$ with duration was present on the external phonocardiogram. Whether in certain patients the early diastolic gradient and concomitant rumble can be of significant magnitude and intensity to transmit to the chest wall is not known at this time.

\section{References}

ALVARES, R.F. (1980). Diastolic Function and Prognosis in Hypertrophic Cardiomyopathy (Doctoral dissertation). London: University of London.

ALVARES, R.F., SHAVER, J.A., GAMBLE, W.H. \& GOODWIN, J.F. (1984). Isovolumic relaxation period in hypertrophic cardiomyopathy. Journal of the American College of Cardiology, 3, 71.

BRAUNWALD, E., LAMBREW, C.T., ROCKOFF, S.D., ROSS, J.JR. \& MORROW, A.G. (1964). Idiopathic hypertrophic subaortic stenosis. Circulation, 29-30: IV-3.

DOI, Y.L., MCKENNA, W.J., GEHRKE, J., OAKLEY, C.M. \& GOODWIN, J.F. (1980). M-mode echocardiography in hypertrophic cardiomyopathy: diagnostic criteria and prediction of obstruction. American Journal of Cardiology, $45,6$.

GLANCY, D.L., SHEPHERD, R.L., BEISER, G. \& EPSTEIN, S.E. (1971). The dynamic nature of left ventricular outflow obstruction in idiopathic hypertrophic subaortic stenosis. Annals of Internal Medicine, 75, 589.

GOODWIN, J.F. (1982). The frontiers of cardiomyopathy. British Heart Journal, 48, 1.

MURGO, J.P., ALTER, B.R., DORETHY, J.F., ALTOBELLI, S.A. \& MCGRANAHAN, G.M. JR. (1980). Dynamics of left
However, regardless of its mechanism of production, it is important for the clinician to appreciate that an early diastolic rumble can occasionally be found in HCM and when present, the clinical presentation may simulate mitral stenosis, particularly when the patient is in atrial fibrillation.

In this review, we have demonstrated that phonoechocardiography can be of great value in accurately displaying the physical findings and anatomical abnormalities of patients with HCM. It is the technique of choice in the initial evaluation of patients with this disease, and in almost all cases it allows the clinician to make a definite diagnosis of this entity.

\section{Acknowledgement}

The authors wish to thank Ms Vicki Shidel for secretarial assistance in the preparation of this manuscript.

ventricular ejection in obstructive and nonobstructive hypertrophic cardiomyopathy. Journal of Clinical Investigation, 66, 1369.

POLLICK, C., RAKOWSKI, H. \& WIGLE, E.D. (1984). Muscular subaortic stenosis: the quantitative relationship between systolic anterior motion and the pressure gradient. Circulation, 69, 43.

REDDY, P.S., SHAVER, J.A. \& LEONARD, J.J. (1971). Cardiac systolic murmurs: pathophysiology and differential diagnosis. In Pathophysiology and differential Diagnosis in Cardiovascular Disease, Friedberg, C.K. and Donoso, E. (eds). New York: Grune \& Stratton.

SHABETAI, R. \& DAVIDSON, S. (1972). Asymmetrical hypertrophic cardiomyopathy simulating mitral stenosis. Circulation, 15, 37.

SHAVER, J. (1981). Current uses of phonocardiography in clinical practice. In Cardiology Update, Rapaport, E. (ed). p. 327.

SMITH, M.R., AGRUSS, N.S., LEVENSON, N.I. \& ADOLPH, R.J. (1975). Nonobstructive hypertrophic cardiomyopathy mimicking mitral stenosis. American Journal of Cardiology, 35, 89. 